\title{
Concept Lattice-Based Semantic Web Service Ontology Merging
}

\author{
Hong Xia \\ Department of Computer Science and Technology, Xi'an University of Posts and Telecommunications, Xi'an 710121, china \\ xiahong@xupt.edu.cn
}

\begin{abstract}
Using ontology semantically express the service of capabilities, correctly match, discovery and composition service. Domain ontology and Formal Concept Analysis aim at modeling concept. In ontology engineering the FCA role is reusing independently developed domain ontology. The method is intended to support the ontology engineer in difficult activities such as ontology merging in the development of the Semantic Web.

Index Terms - Semantic Web Service, concept lattice, Ontology merging
\end{abstract}

\section{I . Introduction}

Semantic Web extends the current Web in information, aims to add machine-interpreted information to Web in order to provide intelligent access to heterogeneous and distributed information. Ontology concepts are extracted the relative concepts in specify domain and used to define semantic Web service. If we could develop ontology which could be used for multiple systems, then shared and reused a common terminology. So support merging ontology which sharing would be possible even between Web services based on different ontology.

In order to solve the problems we involved in our previous work [1], identify possible relations between pairs of Web services by checking semantic similarities. Using an ontology, we can discover relations between two services even the conditions don't match each other syntactically.

Both domain ontology and concept lattice aim at modeling concepts. In this paper, provides a method supports the ontology engineer in reusing existing ontology relying on concept lattice. From a theoretical point of view, ontology concepts are identified with FCA concepts [2]. The main contribution of our method is the possibility of using concept lattice theory to deal with the generating ontology. The rest of the paper is organized as follows. Section 2 introduction related useful results; section 3 shows basic concept including concept lattices and domain ontology, section 4 giving ontology merging algorithm and example; finally section 5 provides the conclusions.

\section{Related Work}

Many researchers consider ontology is a theory of content referring to object types, properties and possible relationships in a specified knowledge domain [3]. In document [4], ontology is the explicit formal specification of items in a domain and the relationships among them. If ontology could be used to the elementary for multiple Web services' semantic information, they would share common terms and facilitate to share and reuse. We provide a method to support merging ontology which sharing would be possible.

In reference [5,6], the importance of dealing with semantically heterogeneous data by using ontology has been emphasized. In reference [6], the methods and tools supporting ontology integration and maintenance can be divided based on Galois Lattices and Description Logics (DL). Reasoning is generally used in order to compute relations among different information sources [7]. In document [8], an ontology merging method based on similarity relations among concepts represented according to DL. The existing work concerning the combination of domain ontology and concept lattice techniques, one proposal concerning a similarity measure for Concept Lattices will be recalled $[9,10]$.

Formal concept analysis $[11,12]$ is proposed by Wille $\mathrm{R}$ in 1982. Concept lattice that is an effective approach to analyze data so has rapidly developed. As formalized tool of data analyzing and knowledge processing, concept lattice theory has been successfully applied to various fields [13,14].

Ontology merging that consists in taking two or more source ontology and returning a merged ontology based on the given source has been investigated in reference [14, 15]. Given two or more source ontology, one context is constructed for each of them, by applying natural language processing techniques.

\section{Basic concepts}

For given service information table $K=(S, A, R)$, is called a formal context in formal concept analysis. As table 1 shows, $S$ is a set of services, $A$ is a set of attributes, $R$ is a binary relation between $S$ and $A, R \in S \times A$. For service $s \in S$, attribute $a \in A$, so $s R a$ express service $s$ has attribute $a$.

TABLE I example of formal concept

\begin{tabular}{|c|c|c|c|c|}
\hline $\mathrm{S} / \mathrm{A}$ & $\mathrm{TA}_{1}$ & $\mathrm{TA}_{2}$ & $\mathrm{TA}_{3}$ & $\mathrm{TA}_{4}$ \\
\hline $\mathrm{TS}_{1}$ & $\times$ & $\times$ & & $\times$ \\
\hline $\mathrm{TS}_{2}$ & $\times$ & & $\times$ & \\
\hline $\mathrm{TS}_{3}$ & & $\times$ & $\times$ & \\
\hline $\mathrm{TS}_{4}$ & $\times$ & $\times$ & & $\times$ \\
\hline $\mathrm{TS}_{5}$ & $\times$ & & & \\
\hline
\end{tabular}

In formal context, for set of service $S$ and set of attribute $A$ may define the following two function $f$ and $g$. 
$\forall S C \subseteq S: f(S C)=\{a \in A \mid \forall s \in S C, s R a\}$

$\forall P C \subseteq A: g(P C)=\{s \in S \mid \forall a \in P C, s R a\}$

$S C$ expresses service class, $P C$ expresses attribute class, $s R a$ expresses existed relation between $s \in S$ and $a \in A$.

From formal context gained each two - tuples (SC, $P C$ ) satisfied $S C=g(P C)$ and $P C=f(S C)$ is defined as formal concept.

Concept lattice $L(K)$ corresponded the formal context $K=(S, A, R)$ is shown in Figure 1.

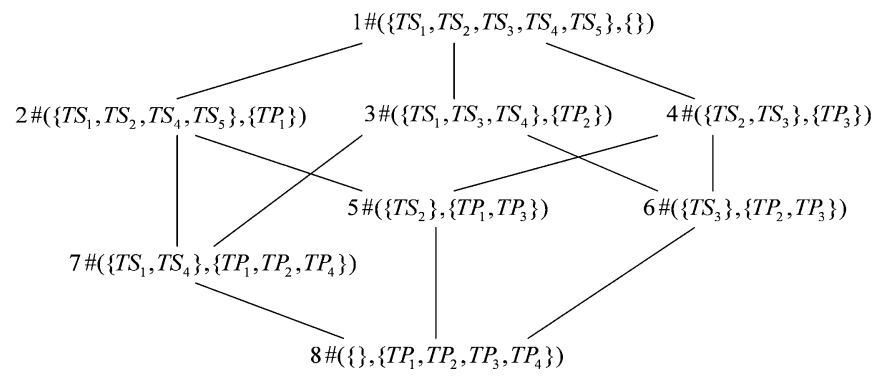

Fig. 1 formal context corresponded the concept lattice

Because concept lattice is represent formal of relationship of between concepts in formal context, it and correspond formal context are one to one correspondence. Therefore, concept lattice distributed processing should involve in divide or merge and so on process about formal context. According to Wille R's idea [12] and reference [16], we have several definitions as follows.

\section{Definition 1}

$K=(S, A, R), K_{1}=\left(S_{1}, A_{1}, R_{1}\right), K_{2}=\left(S_{2}, A_{2}, R_{2}\right), \cdots, K_{i}=\left(S_{i}, A_{i}, R_{i}\right), \cdots, K_{n}=\left(S_{n}, A_{n}, R_{n}\right)$ are called formal context.

(1) if $A_{1}=A_{2}=\cdots=A_{i}=\cdots=A_{n}=A$, then $K_{1}, K_{2} \cdots, K_{i}, \cdots, K_{n}$ 's horizontal union is denoted as:

$K_{1}+K_{2}+\cdots+K_{i}+\cdots+K_{n}=\left(S_{1} \cup S_{2} \cup \cdots \cup S_{i}\right.$

$\left.\cup \cdots \cup S_{n}, A, R_{1} \cup R_{2} \cup \cdots \cup R_{i} \cup \cdots \cup R_{n}\right)$

(2) if $S_{1}=S_{2}=\cdots=S_{i}=\cdots=S_{n}=S$, then $K_{1}, K_{2} \cdots, K_{i}, \cdots, K_{n}$ 's

portrait union is denoted as:

$K_{1} \oplus K_{2} \oplus \cdots \oplus K_{i} \oplus \cdots \oplus K_{n}=\left(S, A_{1} \cup A_{2} \cup \cdots\right.$

$\left.\cup A_{i} \cup \cdots \cup A_{n}, R_{1} \cup R_{2} \cup \cdots \cup R_{i} \cup \cdots \cup R_{n}\right)$.

This definition is that multiple formal context's horizaontal union and portrait union.

Definition $2 K_{i}=\left(S_{i}, A_{i}, R_{i}\right)$ and $K_{j}=\left(S_{j}, A_{j}, R_{j}\right)$ are two different formal contexts in same service domain,
$K_{i} \pm K_{j}=\left(S_{i} \cap S_{j}, A_{i} \cup A_{j}, R_{i} \cup R_{j}\right) \quad$ called two formal contexts' portrait add operation $( \pm)$.

Definition 3 In $K=(S, A, R)$, formal concept $C_{i}=\left(S C_{i}, P C_{i}\right)$ and $C_{j}=\left(S C_{j}, P C_{j}\right)(i \neq j)$

(1) if $S C_{i}=S C_{j}$ then called $C_{i}=C_{j}$, that is concept equal;

(2) if $S C_{i} \supset S C_{j}$ then called $C_{i}$ more than $C_{j}$;

(3) if $S C_{k}=S C_{i} \cap S C_{j}, P C_{k}=P C_{i} \cup P C_{j}$, then $C_{k}=\left(S C_{k}, P C_{k}\right)=C_{i} \pm C_{j}$, that is portrait add operation between concepts.

Definition 4 if $L\left(K_{i}\right)$ and $L\left(K_{j}\right)$ are two intension consistent concept lattice, suppose their portrait union operation $L\left(K_{i}\right) \tilde{\cup} L\left(K_{j}\right)$ equal concept lattice $L(K)$.

Theorem 1 if $L\left(K_{i}\right)$ and $L\left(K_{j}\right)$ are intension consistent concept lattice, then $L\left(K_{i}\right) \tilde{u} L\left(K_{j}\right)=L\left(K_{i} \pm K_{j}\right)$.

Theorem 1 proof sees reference [16].

Concept lattice portrait union algorithm utilize attributebased incremental formation algorithm. Add concept $C=(S C, P C)$ in concept lattice $L(K)$, first in lattice according to the relation of all node and new increase concept, find required modify concept, when relationship between concepts change, corresponding edge will modify.

For concept $C$, its intension and extension respectively are denoted Intension $(C)$ and Extension $(C)$.

Definition 5 for concept $C=(S C, P C)$, if in concept lattice $L(K)$ existed one concept $C_{1}=\left(S C_{1}, P C_{1}\right)$ satisfied $S C_{1} \subseteq$ Extension $(C)$. Concept $C_{1}$ is update concept of $C$. After update it is $\left(S C_{1}\right.$, Intension $\left.(C) \cup P C_{1}\right)$.

Definition 6 for concept $C=(S C, P C)$, if concept lattice $L(K)$ existed one concept $C_{1}=\left(S C_{1}, P C_{1}\right)$, new increase concept $C_{\text {new }}=\left(S C_{\text {new }}, P C_{\text {new }}\right)$ and satisfied:

(1) $S C_{\text {new }}=$ Extension $(C) \cap S C_{1}$ and in lattice does not exist arbitrary concept $C_{2}$ which comes $\operatorname{Extension}\left(C_{2}\right)=S C_{n e w}$ into existence.

(2) arbitrary sub concept $C_{3}$ of concept $C_{1}$, does not exist Extension $\left(C_{3}\right) \cap$ Extension $(C)=S C_{n e w}$, that called concept $C_{1}$ and concept $C$ are generation sub concept of new increase concept $C_{\text {new }}$.

Theorem 2 in concept lattice $L(K)$, if $C_{1}=\left(S C_{1}, P C_{1}\right)$ and $C=(S C, P C)$ are generation sub concept of new increase concept $C_{n e w}=\left(\right.$ Extension $(C) \cap S C_{1}$, Intension $\left.(C) \cup P C_{1}\right)$ 
Theorem 3 supposed in formerly concept lattice $L\left(K_{i}\right)$ and $L\left(K_{j}\right)$, concept ordered by extension's power from little to great. If concept $C_{i}$ of concept lattice $L\left(K_{i}\right)$ is update concept or increase concept of concept $C$ in correspond $L\left(K_{j}\right)$, concept $C^{\prime}$ of $L\left(K_{j}\right)$ insert into $L\left(K_{i}\right)$ after concept $C$, thus does not need consider concept operation between $C^{\prime}$ and concept $C_{i}$.

\section{Algorithm}

Portrait Union Algorithm of Multiple Concept Lattices pseudo-code description as following:

INPUT: $L\left(K_{1}\right), L\left(K_{2}\right), \cdots, L\left(K_{n}\right)$

OUTPUT: $L\left(K_{1}\right) \tilde{\cup} L\left(K_{2}\right) \tilde{\cup} \ldots \tilde{\cup} L\left(K_{n}\right)$.

BEGIN

FOR in $L\left(K_{i}\right)$ concept ordered by extension's power from little to great, $i=2, \cdots, n \mathrm{DO}$

Adopt attribute-based concept generation algorithm, insert concept $(S C, P C) \in L\left(K_{i}\right)$ into $L\left(K_{i}\right)$

\section{ENDFOR}

END

Attribute-based concept lattice incremental generation algorithm:

INPUT: concept lattice $L\left(K_{i}\right)$ and concept $(S C, P C)$

OUTPUT: new concept lattice $L\left(K_{\text {new }}\right)$

BEGIN

FOR each concept node $\left(S C_{i}, P C_{i}\right) \in L\left(K_{i}\right)$ sort ascending by $\left|S C_{i}\right|$ DO

IF node $\left(S C_{i} P C_{i}\right)$ update THEN CONTINUE ENDIF

IF $S C_{i} \subseteq S C$ THEN /*update concept*/

Add $P C$ to $P C_{i}, P C_{i}=P C_{i} \cup P C$;

Insert $\left(S C_{i} P C_{i}\right)$ into VISITED.CS

Set $\left(S C_{i} P C_{i}\right)$ node update or new add tag

IF $S C_{i}=S C$ THEN exit ENDIF

ELSE

$S C_{n e w}=S C_{i} \cap S C ; / *$ generation sub concept $* /$

IF does not exist some $\left(S C_{i}^{\prime}, P C_{i}^{\prime}\right) \in$ VISITED.CS which satisfies $S C_{i}^{\prime}=S C_{\text {new }}$ THEN creates a new node $C_{\text {new }}=\left(S C_{\text {new }}, P C_{1} \cup P C\right)$;

Add edge $\left(S C_{i}, P C_{i}\right) \rightarrow C_{\text {new }}$;

FOR each node $C_{a}$ VISITED.CS DO

IF Extension $\left(C_{a}\right)=S C_{\text {new }}$ THEN

Child:=true;

FOR $C_{a}$ 's every parent node $C_{p}$ DO

IF Extension $\left(C_{p}\right)=S C_{n e w}$ THEN child:=false;
Break;

ENDIF

ENDFOR

\section{IF child THEN}

IF $C_{a}$ is child node of $\left(S C_{i}, P C_{i}\right)$ THEN delete edge $\left(S C_{i}, P C_{i}\right) \rightarrow C_{a}$

ENDIF

Add edge $C_{n e w} \rightarrow C_{a} / * C_{a}$ is immediate child concept of new increase node $* /$

ENDIF

ENDIF

ENDFOR

Add $C_{\text {new }}$ into VISITED.CS

Set $C_{\text {new }}$ node update tag

ENDIF

ENDIF

ENDFOR

END

Give a demonstration of the algorithm.

Divide formal context shown in table1 into two sub context $O_{1}=\left(S_{1}, P_{1}, R_{1}\right)$ and $O_{2}=\left(S_{2}, P_{2}, R_{2}\right)$, where $P_{1}=\left(T P_{1}, T P_{2}\right)$ and $P_{2}=\left(T P_{3}, T P_{4}\right)$. Corresponding sub lattice are $L\left(O_{1}\right)$ and $L\left(O_{2}\right)$, respectively shown in figure 2 and figure 3.

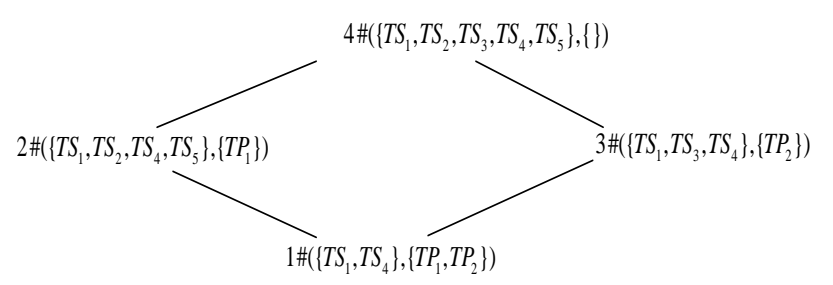

Fig. 2 formal context corresponded the concept lattice $L\left(O_{1}\right)$

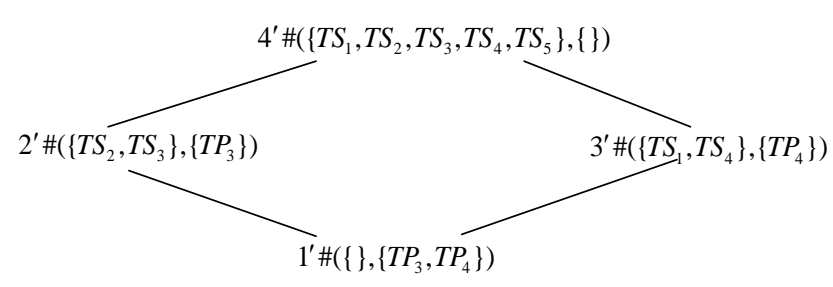

Fig. 3 formal context corresponded the concept lattice $L\left(O_{2}\right)$

Now add nodes of $L\left(O_{2}\right)$ to $L\left(O_{1}\right)$ in turn.

Step 1 add node $\# 1^{\prime}$ : First compute $\# 1,\{\} \cap\left\{T S_{1}, T S_{4}\right\}=\{\}$, $\left\{T P_{1}, T P_{2}\right\} \cup\left\{T P_{3}, T P_{4}\right\}=\left\{T P_{1}, T P_{2}, T P_{3}, T P_{4}\right\} \quad, \quad$ gain node $\# 5\left(\{\},\left\{T P_{1}, T P_{2}, T P_{3}, T P_{4}\right\}\right)$, in $L\left(O_{1}\right)$ and $L\left(O_{2}\right)$ there does not exist node less than $\# 1$ and $\# 1^{\prime}$, so node $\# 5\left(\{\},\left\{T P_{1}, T P_{2}, T P_{3}, T P_{4}\right\}\right)$ is new increase node, its generator sub formula is \#1; \#1' and \#1 's next node generation extension 
like \#5, thus does not generate new increase node, after add $\# 1^{\prime}$, lattice shown in figure 4 , where overstriking node is new increase node, overstriking real line denotes of connection between it and its generator sub node.

Step 2 add node \#2' : it adds after node $\# 1^{\prime}$ adds, thus does not consider operation between it and new increase node \#5. Compute with node $\# 1$, because $\left\{T S_{1}, T S_{4}\right\} \cap\left\{T S_{2}, T S_{3}\right\}=\{\}$, does not generate new node, compute \#2 $\left\{T S_{1}, T S_{2}, T S_{4}, T S_{5}\right\} \cap\left\{T S_{2}, T S_{3}\right\}=\left\{T S_{2}\right\},\left\{T P_{1}\right\} \cup\left\{T P_{3}\right\}=\left\{T P_{1}, T P_{3}\right\}$, gain node $\# 6\left(\left\{T S_{2}\right\},\left\{T P, T P_{3}\right\}\right)$. In $L\left(O_{1}\right)$ and $L\left(O_{2}\right)$ nodes less than \#2 and less than \#2' have not equal or more than \#6, so node $\# 6\left(\left\{T S_{2}\right\},\left\{T P_{1}, T P_{3}\right\}\right)$ is new increase node. Its generate sub formula is \#2, by the same way, compute with node $\# 3$ and generate new increase node $\# 7\left(\left\{T S_{3}\right\},\left\{T P_{2}, T P_{3}\right\}\right)$. Compute with node \#4 and generate new increase node $\# 8\left(\left\{T S_{2}, T S_{3}\right\},\left\{T P_{3}\right\}\right)$, here new increase nodes which express add to $L\left(O_{1}\right)$, after add \#2' lattice shown in figure 5 .

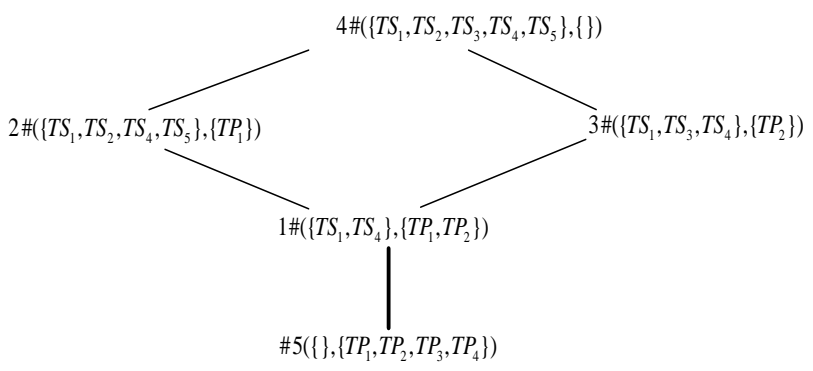

Fig. 4 add node \# $1^{\prime}$ of $L\left(O_{2}\right)$ in $L\left(O_{1}\right), L\left(O_{1}\right)$ change sketch map

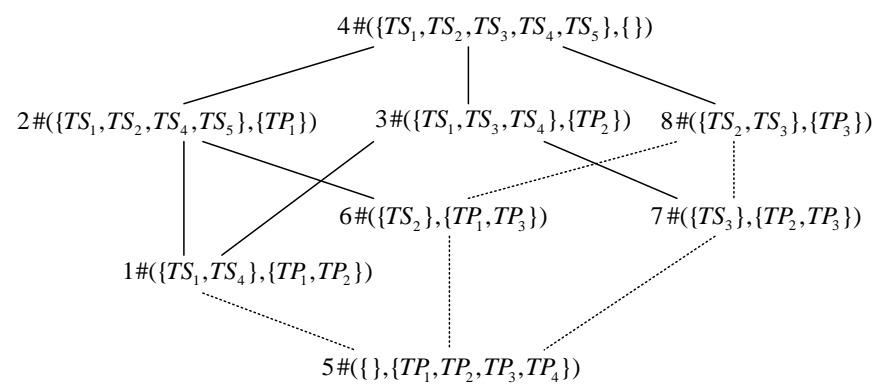

Fig. 5 add node \#2' of $L\left(O_{2}\right)$ in $L\left(O_{1}\right), L\left(O_{1}\right)$ change sketch map

Step3 add node \#3' : because does not consider operation between it and new increase node, first compute with node\#1, because $\left\{T S_{1}, T S_{4}\right\} \subseteq\left\{T S_{1}, T S_{4}\right\}$, node \#1 needs update, forms update $\left(\left\{T S_{1}, T S_{4}\right\},\left\{T P_{1}, T P_{2}\right\} \cup\left\{T P_{4}\right\}\right)=\left(\left\{T P_{1}, T P_{4}\right\},\left\{T P_{1}, T P_{2}, T P_{4}\right\}\right) ; \# 3^{\prime}$ and later \#2, \#3 and \#4 operation form extension $\left\{T S_{1}, T S_{4}\right\}$, thus does not process again, after add \#3', lattice actually same as figure 1 .

Step 4 add node $\# 4^{\prime}$ : only consider it operation with node \#2, \#3 and \#4 . Obviously, node \#2, \#3 and \#4 need update, but node \#4' intension is empty, so these node do not change. It is observed that $L\left(O_{1}\right) \tilde{\cup} L\left(O_{2}\right)=L\left(O_{1} \pm O_{2}\right)=L(O)$

\section{Conclusions}

In this paper, we have invested the problem of constructing and merging ontology of Web service using concept lattice theory. We form concept lattice and have a novel view to solution the service ontology constructing. And then we conclude the study as a Portrait Union of Multiple Concept lattices algorithm based on the discussion before. A merge example has illustrated the algorithm. Our future work is constructing actual service ontology based on concept and extending the proposed results to another research problem .

\section{Acknowledgment}

The research is sponsored by Shaanxi Provincial Department of Education Project (11JK1042).

\section{References}

[1] X. Hong, L. Zeng-zhi, and C. Yan-ping. Research of semantic web service matching based on concept lattice. Journal of Beijing University of Posts and Telecommunications, 29(5):185-188, May 2006.

[2] M. Bain, Inductive construction of ontologies from Formal Concept Analysis, Australian Conference on Artificial Intelligence, 2003, pp.8899.

[3] B.Chandrasekaran, J. Josephson, and V. Benjamins. What are ontologies and why do we need them? IEEE Intelligent Systems, pages 20-26, Jan/Feb 1999.

[4] T. Gruber. A translation approach to portable ontology specification. Knowledge Acquisition, 5(2):199-220, 1993.

[5] N. Lammari, E. Metais, Building and maintaining ontologies: a set of algorithms, Data and Knowledge Engineering, 48(2), pp.155-176, 2004.

[6] G. Pierra, The PLIB ontology-based approach to data integration, IFIP Congress Topical Sessions, 2004, pp.13-18.

[7] D.Calvanese, G. De Giacomo, M. Lenzerini, A framework for ontology integration, in: Proceedings of Semantic Web Working Symposium (SWWS),2001,pp.303-316.

[8] F. Hakimpour, A. Geppert, Resolving semantic heterogeneity in schema integration, International Conference on Formal Ontology in Information Systems (FOIS), 2001,pp.297-308.

[9] R.Belohlavek, Combination of knowledge in fuzzy Concept Lattices, International Journal of Knowledge-Based Intelligent Engineering Systems, 6(1), pp.9-14, 2002.

[10] R.Belohlavek, J.Dvorak, J. Outrata, Fast factorization of Concept Lattices by similarity: solution and an open problem, in: V. Snasel, R.Belohlavek (Eds.), Proceedings of Concept Lattices and their Applications (CLA), Ostrava, Czech Republic, 2004, pp.47-57.

[11] R. Wille. Restructuring lattice theory: an approach based on hierarchies of concepts. In I. Rival, editor, Ordered Sets, pages 445-470, DordrechtBoston, 1982. Reidel Publishing Company.

[12] B. Ganter and R. Wille. Formal concept analysis, mathematical foundations. Spring-Verlag, Berlin Heidelberg, 1999.

[13] W. Petersen,"A Set-Theoretical Approach for the induction of Inheritance Hierarchies," Theoretical Computer Science, vol. 53, pp.296-308, 2004.

[14] G. Stumme and A. Maedche, "FCA-Merge:Bottom-Up Merging of Ontologies", Proc. 17th Int'1 Conf. Artificial Intelligence (IJCAI'01), pp.225-230,2001.

[15] G. Stumme, R. Taouil, Y. Bastide, N. Pasquier, and L. Lakhan, "Computing Iceberg Concept Lattice with Titanic," J. Knowledge and Data Eng., vol. 42, no.2, pp. 189-222,2002.

[16] L. Yun, L. Zong-tian, C. Ling, X. Xiao-hua, and C. Wei. Horizontal union algorithm of multiple concept lattices. ACTA ELECTRONICA SINICA, 32(11):1849-1854, 2004. 